\title{
Research on adaptive selection and allocation mechanism of cloud manufacturing service based on semantic
}

\author{
TAI Li-jun ${ }^{1, a}$, HU Ru-fu ${ }^{2, b}$, CHEN Cao-wei ${ }^{3, c}$ \\ ${ }^{1}$ school of mechanical engineering, Ningbo University of Technology, china \\ ${ }^{2}$ school of mechanical engineering, Ningbo University of Technology, china \\ ${ }^{3}$ school of mechanical engineering, Ningbo University of Technology, china

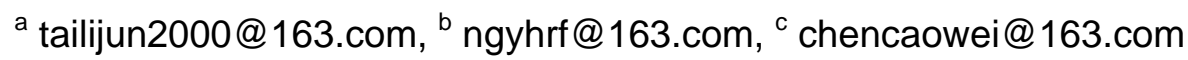

Keywords:cloud manufacturing, ontology,semantic

\begin{abstract}
In this paper domain ontology model of cloud manufacturing service and suitable semantic description scheme of manufacturing service was study, the method of dynamic perception and effectively cognition of the manufacturing resources was study.To solve the integration, sharing, virtual key problems in the application of cloud manufacturing,to promote the application of cloud manufacturing technology ,such as dynamic perception and effective cognitive of manufacturing resources in practice.
\end{abstract}

\section{Introduction}

Cooperation and coordination of inter- organization has become the theme of development of Modern manufacturing enterprises in globalization economic. Over the past 20 years under the guidance of advanced manufacturing concepts and ideology,such as agile manufacturing, network manufacturing, manufacturing oriented service ${ }^{[1.2]}$, many domestic and foreign research institutions carried out a lot of research and practice on the virtual enterprise, collaborative network, manufacturing grid, application service providers, cloud manufacturing service mode. Some achievements have been obtained and implements some typical applications ${ }^{[3,4]}$. Among them the practice and application of cloud manufacturing service model will become important supporting means of China's manufacturing enterprises to fully utilize and share manufacturing resources, improve product design, management and manufacturing capacity, enhance the comprehensive competitiveness of enterprises, It is also an important development direction of exploration of the current advanced manufacturing field in our country ${ }^{[5]}$.

The related research of cloud manufacturing is still in the initial stage. The research mainly concentrated on the core theory, technology platform standard, specification and platform architecture etc.There are many aspects need to further study for the specific implementation technology and application.

In this paper based on the analysis of composition of cloud manufacturing technology and the characteristics of virtual manufacturing resource allocation in cloud manufacturing service.

Domain ontology model of cloud manufacturing service and suitable semantic description scheme of manufacturing service was study, the method of dynamic perception and effectively cognition of the manufacturing resources was study.

To solve the integration, sharing, virtual key problems in the application of cloud manufacturing,to promote the application of cloud manufacturing technology ,such as dynamic perception and effective cognitive of manufacturing resources in practice.

\section{Construction of the ontology model and semantic description scheme of manufacturing service}

According to the organization way of ontology, at present it can be divided into the single ontology, Multiple ontologies and hybrid ontology. 
The application of cloud manufacturing service itself appears polymorphism, And it is difficult to construct single ontology, different application industries have different perspectives and different requirements, so multilayer ontology structure is very suitable for constructing cloud manufacturing service ontology. Therefore, the following three layers of cloud manufacturing service ontology model was build, as shown in figure 1:

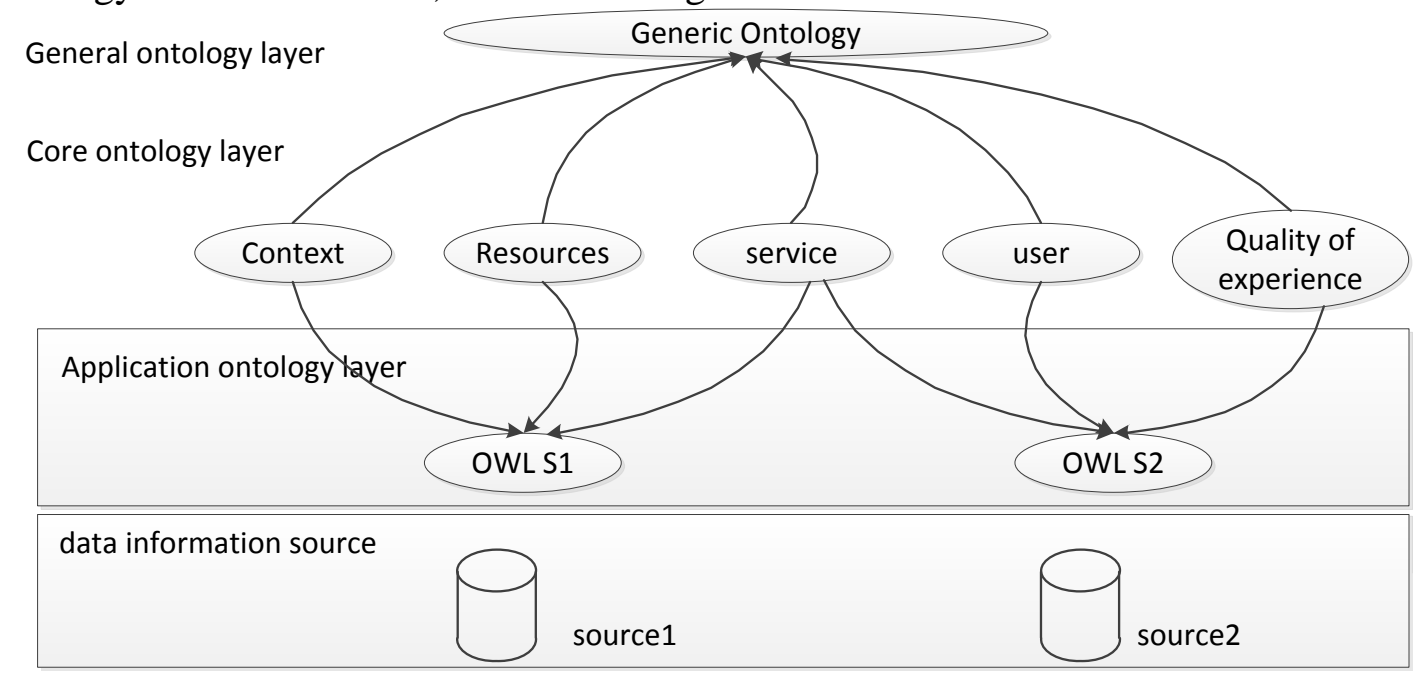

Fig. 1 ontology structure model of cloud manufacturing service

Currently most of the web services are described based on the WSDL language. However, WSDL only supports at operation of the syntax level, lack of semantic expression of web service ability . And the cloud manufacturing service have an urgent demand on semantic, so we need to choose services language support semantic description. Web service markup language OWL-S (formerly DAML-S), is a kind of service description ontology model which is widely used, semantically rich, lightweight, easy to expand. OWL-S provides a method for describing the service and the corresponding functional attributes.

As shown in Figure 2,the characteristics of a service is descripted by three top-level concept of resource ,as service profile, service process model, Service base. Therefore, this paper aims to select the OWL-S language to describe the ontology of cloud manufacturing service model.

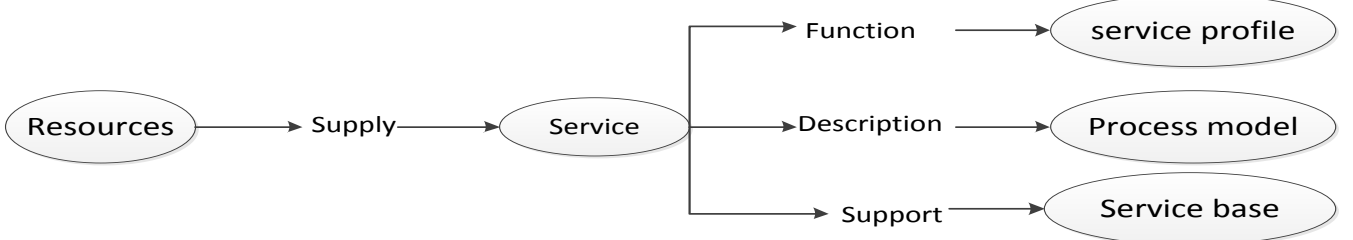

Fig. 2 OWL- S top level ontology structure

\section{Dynamic perception and effectively cognition of manufacturing resources}

Dynamic perception and validity of cognition of Manufacturing resource needs to solve the key problems of construction of dynamic sensing environment, definition of model and the establishment of composite frame etc.

Dynamic sensing environment constructed by the following methods: 1)dispose various sensing devices on the manufacturing resources (such as thermal, speed, vibration sensor) to apperceive various state parameters of manufacturing resources, and to realize intelligent sensor, signal acquisition and intelligent processing combining intelligent embedded technology; (2)dispose the RFID reader on the manufacturing equipment, bind RFID labels on articles being processed and materials, and use manufacturing execution system based on RFID to realize the recognition of manufacturing resources,automatic acquisition of information and monitoring and scheduling of manufacturing process; (3)use the internet of things access device to realize manufacturing resource network access, and realize transmission and communication of information through the internet; (4) use perception model of manufacturing resources to build the mapping from the entity of 
resources to virtual image; (5) use cognitive models of manufacturing resources to build the mapping from the virtual image to the instant ability; 6)when the real time state of manufacturing resource changes, the virtual image will real-time refresh by using the perceptual model and then with the help of the cognitive model of real-time updates its instant service ability.

\section{Model definition}

Perception model: reflect the virtual image of physical state when manufacturi- ng resources running.

Cognitive model: describe the instant service ability of manufacturing resources.

This paper put forward the following perception model scheme based on the extended study that open FRID public service platform research on code analys and services of discovery, as shown in figure 3:

\section{Summary}

Explore the method of dynamic perception and effectively cognition of the manuf acturing resources. Promot the manufacturing resource dynamic perception and effective cognition of the cloud manufacturing technology using in practical application. To provide reference for chinese manufacturing enterprises in the cloud manufacturing service application.

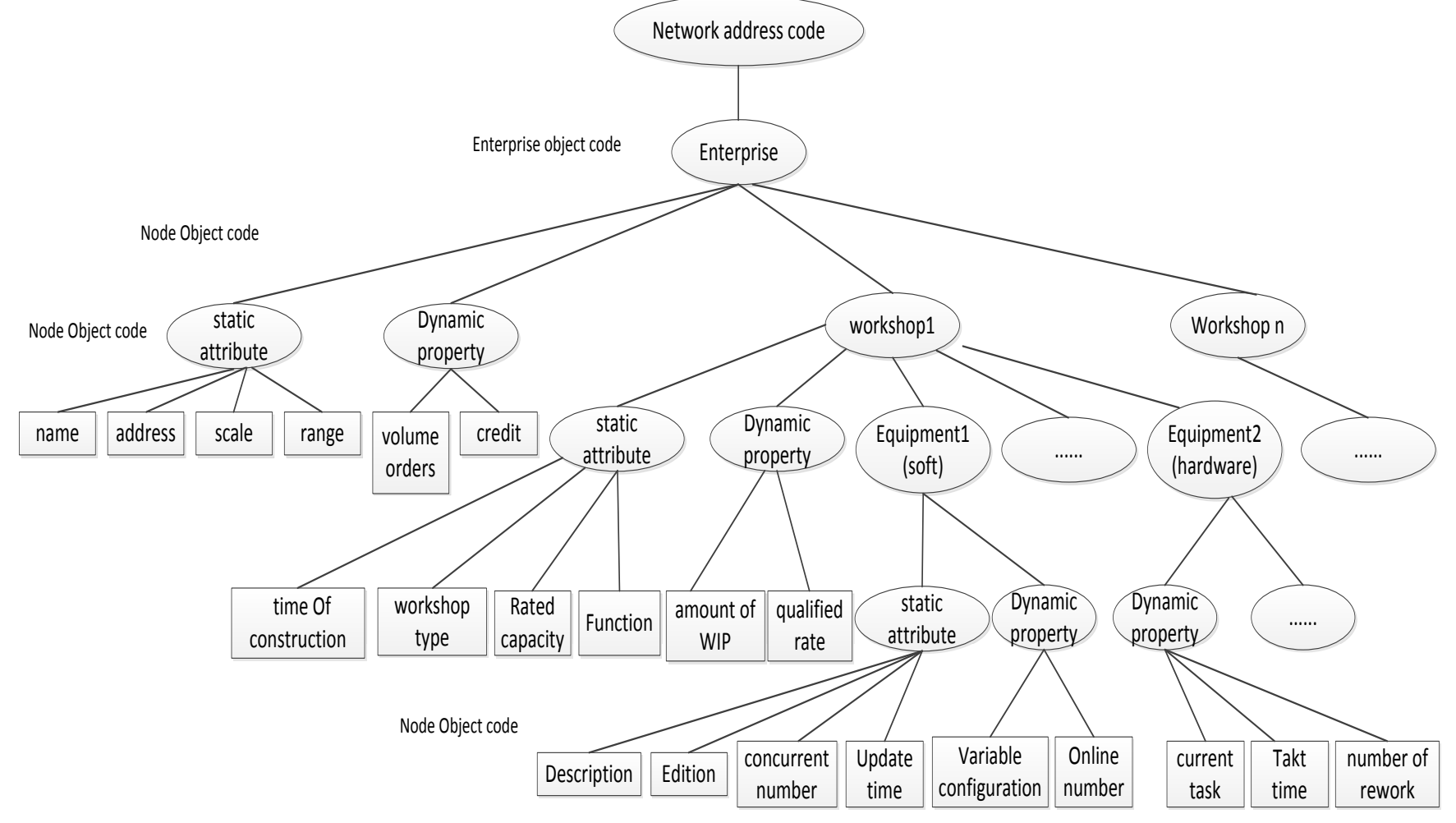

Fig. 3 Perception model scheme

\section{References}

[1] YIN Chao, HUAN Bi-qing, LIU Fei,etc. Common key technology system of cloud manufacturing service platform for small and medium enterprises [J], Computer Integrated Manufacturing Systems, 2011, 17(3): 495-503.

[2] YIN Sheng, YIN Chao, LIU Fei,etc. Outsourcing resources integration service mode and semantic description in Cloud manufacturing environment [J]. Computer Integrated Manufacturing Systems, 2011, 17(3):525-532. 
[3] BLOMQVIST E, HGREN A. Constructing an enterprise ontology for an automotive supplier[J].Engineering Applications of Artificial Intelligence,2008,21(3):386-397.

[4] Resnik P.Using Information Content to Evaluate Semantic Similarity in Taxonomy, Proceedings of the14th International Joint Conference on Artificial Intelligence. Chelm -sford,1995.448 449.

[5] YANG Gang. Matching Technology Research of Semantic Web Services Based on Ontology [D].Nanchang: Jiangxi University of Science and Technology,2009. 